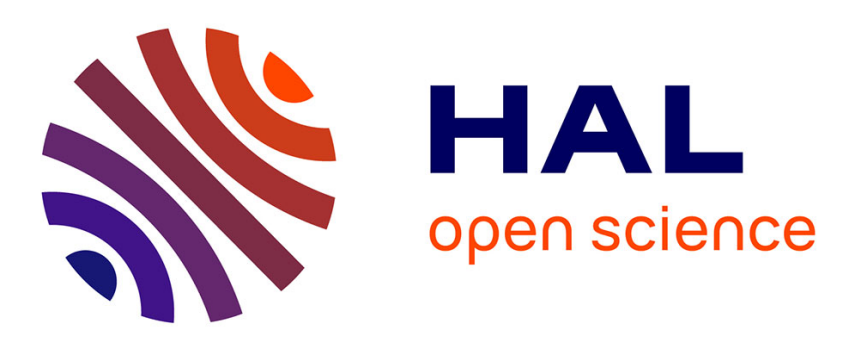

\title{
Influence of the yttria powder morphology on its densification ability
}

Agnès Dupont, Alain Largeteau, Claude Parent, Bruno Le Garrec, Jean-Marc Heintz

\section{- To cite this version:}

Agnès Dupont, Alain Largeteau, Claude Parent, Bruno Le Garrec, Jean-Marc Heintz. Influence of the yttria powder morphology on its densification ability. Journal of the European Ceramic Society, 2005, vol. 25, $\mathrm{n}^{\circ} 12$, p. 2097-2103. 10.1016/j.jeurceramsoc.2005.03.016 . hal-00015501

\section{HAL Id: hal-00015501 https://hal.science/hal-00015501}

Submitted on 8 Dec 2005

HAL is a multi-disciplinary open access archive for the deposit and dissemination of scientific research documents, whether they are published or not. The documents may come from teaching and research institutions in France or abroad, or from public or private research centers.
L'archive ouverte pluridisciplinaire HAL, est destinée au dépôt et à la diffusion de documents scientifiques de niveau recherche, publiés ou non, émanant des établissements d'enseignement et de recherche français ou étrangers, des laboratoires publics ou privés. 


\title{
Dupont Agnès, Largeteau A., Parent Claude, Le Garrec Bruno, Jean-Marc Heintz
}

\begin{abstract}
:
The present paper shows that we can predict the densification capacity of an yttria powder in relation with its grain shape by using a simple thermodynamic model. All other parameters, such as temperature, time, sintering atmosphere and nature of the powder are fixed. The values of the relative density and grain size of the final ceramic strongly depend on the shape of the grain of the initial powder. A simple model is proposed to understand these differences and to give tools to predict, step by step the densification capacity of a powder. This study could be extended to powders of different nature
\end{abstract}

Keywords : Grain growth ; Sintering ; Grain size ; Rare earth oxides

\section{Introduction}

Ceramics have gained importance in electronic as well as optical areas. But for these specific applications, high-density ceramics are required. Thus, increasing more and more the densification capacity of the powders without spending too much energy on heating is today a real challenge.

Understanding more precisely the intrinsic reasons for high densification remains a difficult concern. One of the best ways to avoid too much heating would be to act on the grain shape. Doh-Yeon Kim performed very interesting work ${ }^{1,2}$ and 3 concerning the effect of grain shape on the grain-coarsening behavior during liquid-phase sintering. He explained the reasons for the mechanism to be either diffusion or 2D nucleation during sintering and depending on the grain shape. However, he did not develop the real impact on the final density.

By focusing, for example, on yttria powder as the starting powder, it appears that most of the time, the densification capacity is studied as a function of the time, or the temperature, or more generally the sintering cycle but only for a given morphology. Spheres are often chosen, because they are well known to be a good morphology for sintering. Akinc ${ }^{4}$ proposes pertinent study of monosized spherical yttria powders sintered respectively at 1500,1600 and $1700{ }^{\circ} \mathrm{C}$ for 7 to $720 \mathrm{mn}$ to reach density of $98-99 \%$. Rhodes ${ }^{5}$ shows that the effects of agglomeration size of fine yttria stabilized zirconia limit the green density and also limit final sintering stage to $95 \%$. He also proposes a method ${ }^{6}$ to retard grain growth to get a pore-free ceramic of $\mathrm{La}_{2} \mathrm{O}_{3}-\mathrm{Y}_{2} \mathrm{O}_{3}$ leading to $100 \%$ theoretical density by heating until $2170{ }^{\circ} \mathrm{C}$. Full density is related to high sintering temperatures most of the time.

We propose in this paper, to fix the sintering conditions, such as time, temperature, atmosphere and powder nature to yttria, and to discuss only the influence of the grain shape on the sintering capacity. This study proves that not only the spheres are good candidates for further densification, but also grooved grains, or other massive and regular objects. This study gives tools to predict the sintering capacity of a given powder in relation to its grain shape and these predictions may be extended to other powders.

\section{Experimental procedure}

Yttria powders have been synthesized by a chemical route involving various chelating agents. The synthesis has been described precisely in previous work. ${ }^{\underline{7}}$ In relation to this previous paper, only powders calcined at $1100{ }^{\circ} \mathrm{C}$ have been studied in the present work and they are listed in Table 1 with a brief description of the grain shape. Pellets have been prepared from each powder and they have been isostatically pressed at $200 \mathrm{MPa}$. Then, the resulting pellets are sintered at a rate of $1000^{\circ} \mathrm{C} / \mathrm{h}$ to the desired temperature of $1700^{\circ} \mathrm{C}$, then maintained $3 \mathrm{~h}$ under vacuum of $10^{-5} \mathrm{mbar}$. 
isostatic pressing at $200 \mathrm{MPa}$, sintering was performed in a home-made furnace. The grain morphology was obtained from scanning electronic microscope observations (SEM, JEOL 840) after optical polishing and thermal etching of a ceramic section. The intercept method was used.

\section{Results and discussion}

In this section, the sintering conditions were set to $1700{ }^{\circ} \mathrm{C}$ for $3 \mathrm{~h}$ under vacuum and the values of relative densities and grain size are compared for all yttria pellets. The only differences between the samples are the initial grain morphology of the yttria powder as shown in Fig. 1 . The relative grain densities of the isostatically pressed pellets are presented in Table 1, as well as the relative densities and the grain sizes. What is striking is that the values of relative density and grain size (Table 1 ) depend strongly on the initial grain shape and size, and we propose the following model to explain the differences observed.

First, we focus on the ideal case of spheres as the initial yttria powder grain shape. Fig. 2 gives a representation of the evolution of the energy of the system as a function of the sintering path during the sintering process. As it is well known for spheres, the sintering process could be artificially divided into two distinct steps $^{8}$ : densification and grain growth corresponding to the two bumps on the diagram. We are aware that the phenomena of densification and grain growth may occur partly simultaneously in the real transformation and the representation of two separate steps remains a theoretical view. Second, insofar as the densification rate is an inverse function of the initial sphere size, the smaller the sphere diameter, the quicker the densification step occurs. That is why results obtained with initial sphere size of $200 \mathrm{~nm}$ lead to final grain size of $24.5 \mu \mathrm{m}$ and relative density of $99.2 \%$ which are quite high values according to densification capacity of small spheres.

Let us now focus on the second case illustrated by Fig. 3. We use the term grooved needles to describe a column of several spheres arranged like beads on a string. An example may be seen at the bottom of Table 1 . The initial geometry of the sintering path consists indeed in associated spheres exhibiting preferential interactions along needle axe. ${ }^{7}$ We propose here a three-step sintering mechanism where the first step would correspond to the rearrangement of spheres into a full packing of spheres avoiding macroporosity where as steps 2 and 3 remain densification and grain growth as before. Thus, the first additional step consists in moving spheres in order to fill large pores and allows the system to reach the initial stage of the previous case of spheres. This step of rearrangement during sintering has been shown by H.E. Exner in the case of glass and also cupper spheres, ${ }^{9}$ and 10 where the formation of new contacts between spheres have been observed from 7 contacts corresponding to a green density of $64 \%$ to $13-14$ contacts at full density. Dudrova ${ }^{11}$ also observed the rearrangement of spheres of iron to reach new contacts between particles. Her measurements show angular rotation velocities as high as $0.17 \% / \mathrm{s}$ during heating. It appears then that the grains rotate or twist in response to torques generated by unbalanced surface energies, inhomogeneous packing coordinations and different crystallographic orientations at the contacts. Then, the stage reached at the end of the first step corresponds to the first case of spheres. Thus densification and grain growth are the following steps of sintering. Large relative density of $99.1 \%$ but small grain size of $5.2 \mu \mathrm{m}$ reveal that this reaction did not get enough time to complete the sintering process compared to the first case. This statement may be explained by this three-step model which shows that the rearrangement of spheres into a compact assembly is an additional step requiring free activation energy and time to occur. According to the figure, we have a three-step sintering process. Then, due to this additional step, and due to a sintering time of $3 \mathrm{~h}$ remaining constant for each sample, the time is not sufficient to reach complete sintering. That is why nearly full density is reached but not very large grain size, which happens in the last step.

Finally, Fig. 4 deals with the case of thin and irregular platelet geometry. We propose now a fourstep sintering mechanism with a first additional step of "spheroidization", followed by rearrangement of spheres, densification and grain growth. To confirm this proposition, we propose the following proofs.

1. Insofar as the additional step would occur first, it means that the density is still low like green density of around $50 \%$ and the particles are partly surrounded by gas. The observations of Lange ${ }^{12}$ and 13 related to particles surrounded by gas and presenting large shape factors are applicable to this case: he pointed out that this type of grooved needles when heated allow the system to relax to its lowest energy level. We have observed this phenomenon during calcination for needles ${ }^{7}$ and Fig. 1 a. Given that the diameter of the needles is less than the thickness of the platelets, higher energy is required to spheroidize, and that means, according to Langue's interpretation, higher energy for crystallites to reach the thickness of the platelets. 
2. Doh-Yeon Kim showed also that the $\mathrm{ZnO}$ grains of a characteristic angular shape and surrounded by a liquid phase at low temperature become spherical with the increase of temperature. ${ }^{14}$ The only difference between this description and our case is the liquid phase present whereas we have only gas surrounding the particles. Then the atomic diffusion will be slower than for Kim's experiments but the final state of spherical particles is the same and corresponds to the thermodynamic stable state.

3. This is also proved by the SEM micrograph (Fig. 5) realized in a pore after $3 \mathrm{~h}$ sintering of the platelets. Pores guarantee that the step of densification does not occur and then that only spheroidization (step 1), rearrangement into a close packing as much as possible (step 2 ) and grain growth (step 4) take place. Thus, the memory of the first step of spheroidization is maintained. And that is what we observed by SEM measurements made in the pore of an initial powder composed of platelets that do not easily densify. Particles look like spheres in the pore. This is clearly visible in Fig. 5, again a proof of this step of spheroidization.

4. Insofar as platelets are $500 \mathrm{~nm}$ thick, emerging spheres are larger than for needles where the diameter is only $200 \mathrm{~nm}$. According to the model we proposed, the second step would consist in the rearrangement of large spheres into a compact assembly which avoid macroporosity. During the third step, densification occurs and is higher than for small spheres because the densification rate is an inverse function of the initial grain size. Finally, grain growth occurs. What appears is that neither the density of $93 \%$ nor the grain size of $3 \mu \mathrm{m}$ are large. This result may be simply explained by our model which shows that we have a four-step sintering process: time is needed to make spheres, which is already an additional step compared to the second case related to grooved needles, and time must be again provided to have a dense assembly of spheres. Compared to the first case on nanospheres, these two last steps require additional time to occur. On top of all this, densification is slower because of the large sphere size. That is why there is not enough time to achieve complete sintering and the relative density remains small as well as the grain size.

At this step of the thought process, it appears that a two-, three- or four-step sintering mechanism occurs, depending on the initial grain shape of the powder. The two-step mechanism corresponds only to non-agglomerated spheres, whereas the three-step mechanism consist in particles forming spheres, but developing preferential interactions along many different directions. These systems can be obtained after the calcination of the particles presenting large shape factors as Lange showed. Finally, the four-step sintering mechanism is related to all other geometries which are far away from the ideal case of spheres. Then, if all other parameters are fixed such as time and temperature of sintering, the evolution of relative density and grain size can be predicted in relation with the initial grain powder morphology.

Finally, this model allows us to choose the initial powder morphology in relation with the desired functionality of the ceramic (dense or not). In order to have a dense ceramic, the best solution would consist in a two-step sintering process which is the case only for spheres as initial powder. If a three- or four-step process takes place, the only way to reduce the barrier of energy of the step of rearrangement of spheres involves reducing the macroporosity, which depends strongly on the initial powder morphology. The more regular the particles are, the easier the step of rearrangement will be. Finally, concerning the step of spheroidization, the two ways to reduce the barrier of energy are, on the one hand to have particles already grooved during calcination such as grooved needles, or on the other, to have particles as thin as possible, which leads more easily to spheroidization because crystallites have the same size as particles. As a conclusion, we assume that good sinterable powders consist in small spheres or grooved particles even if they present high shape factors: this result is new as far as we know. Regular grains are less sinterable powders than small spheres and they need higher energy to sinter but can reach full density if enough energy is provided. Finally, irregular particles give poor sinterable powders that will never reach high densities.

\section{Conclusion}

This work aimed at proposing new ways to predict the densification capacity of a powder, only depending on its grain shape, and by using a thermodynamic model. This model allows us to choose the initial powder morphology in relation with the desired functionality of the ceramic such as density. Generally speaking, even if spheres remain a good candidate for highly efficient sintering, other morphologies, such as grooved particles, or very regular particles also display very good densification capacity. Tools have also been proposed to think through, step by step, the influence of the morphology on the sintering time and densification capacity. Results proposed for yttria powders 
may be applicable to other powders nature provided that no other sintering phenomena occur.

\section{Acknowledgments}

This work was sponsored by the Commisariat à l'Energie Atomique and the authors also wish to express their gratitude to Region Aquitaine for its financial support. We would like also to thank sincerely Christian Chatillon and Mathias Hermann from Institut National Polytechnique de Grenoble for effective participation to sintering experiments and Alain Largeteau from Institut de Chimie et de la Matière Condensée de Bordeaux who performed the isostatic pressing of the yttria powder.

\section{References}

1 D-Y. Kim, Conference of the American ceramic society Nashville (2003).

2 M.-K. Kang, D.-Y. Kim and N.M. Hwang, J. Eur. Ceram. Soc. 22 (2002), pp. 603-612.

3 H.R. Lee, D.J. Kim, N.M. Hwang and D.Y. Kim, J. Am. Ceram. Soc. 86 (2003) (1), pp. 152-154.

4 D.J. Sordelet and M. Akinc, J. Am. Ceram. Soc. 71 (1988) (12), pp. 1148-1153.

5 W.H. Rhodes, J. Am. Ceram. Soc. 64 (1981) (1), pp. 19-22.

6 W.H. Rhodes, J. Am. Ceram. Soc. 64 (1981) (1), pp. 13-19.

7 A. Dupont et al., J. Solid State Chem. 171 (2003), pp. 152-160.

8 D. Bernache-Assolant, Chimie-Physique de Frittage, ed. Hermes 1993, p. 179.

9 G. Petzow and H.E. Exner, Z. Metallk. 67 (1976), p. 611.

10 H.E. Exner and G. Petzow, Shrinkage and rearrangement during sintering of glass spheres. In:

G.C. Kuczynski, Editor, Sintering and Catalysis, Plenum Press, New York (1975), p. 279.

11 E. Dudrova, E. Rudnayova and G. Leitner, Powder Met. Interm. 16 (1984), p. 255.

12 K.T. Miller and F.F. Langue, Acta Metallogr. 37 (1989), pp. 1343-1347.

13 K.T. Miller, F.F. Langue and D.B. Marshall, J. Mater. Res. 5 (1990), pp. 151-160.

14 J-H. Han, Y-K. Chung, D-Y. Kim, S-H. Cho and D.N. Yoon, Acta Metall. 37 (1989) (10), pp. 2705-2708. 
Table 1

Values of green densities, relative densities and grain sizes for the sintering conditions of $1700^{\circ} \mathrm{C}$ for $3 \mathrm{~h}$ under vacuum of $10^{-5} \mathrm{mbar}$

SEM of yttria powders

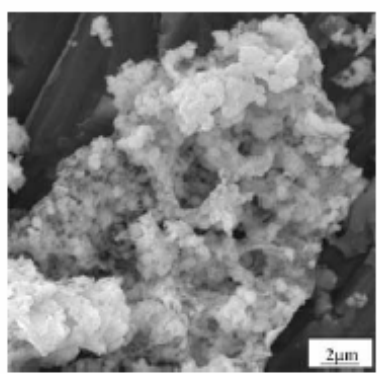

Acetate route at $R=20$

Oxalate route at $R=2$

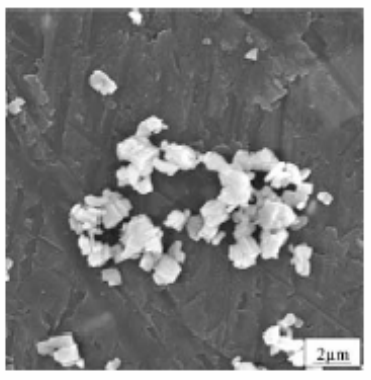

Oxalate route at $R=20$
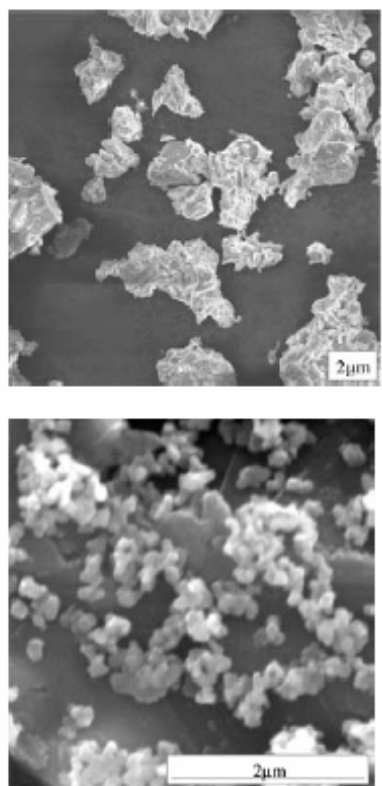

$R=2$
Powder characteristics

\section{SEM of $\mathrm{Y}_{2} \mathrm{O}_{3}$ ceramics}

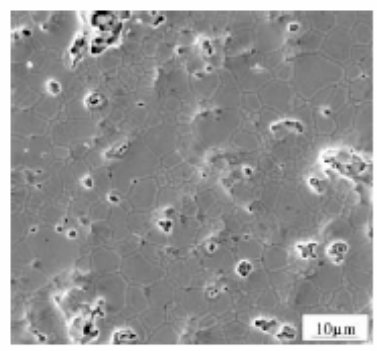

Irregular platelets

Foam made up of spherical grains

Small and thick regular platelets

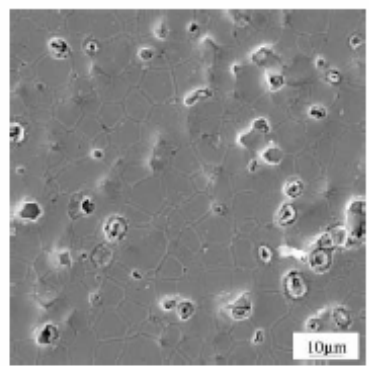

3.5

$95.5 \%, 49.3 \%$

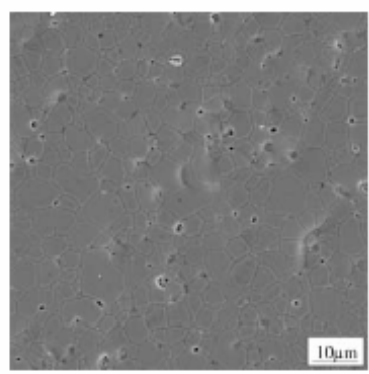

3.0

$96.8 \%, 54.3 \%$

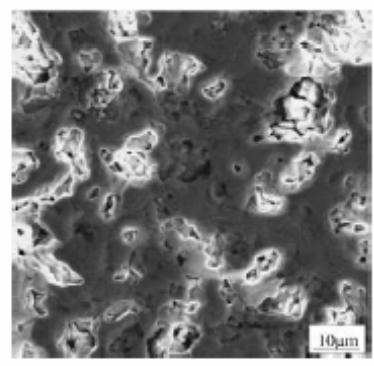

Small spherical and agglomerated grains

Grain size Relative density, $(\mu \mathrm{m})$ Green density

$93.0 \%, 49.5 \%$

Sandstone like powder

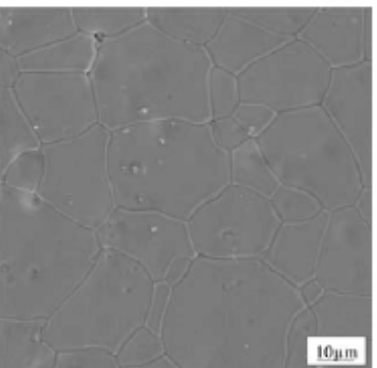

$2.4 \quad 85.0 \%$

$24.5 \quad 99.3 \%, 46.4 \%$ 


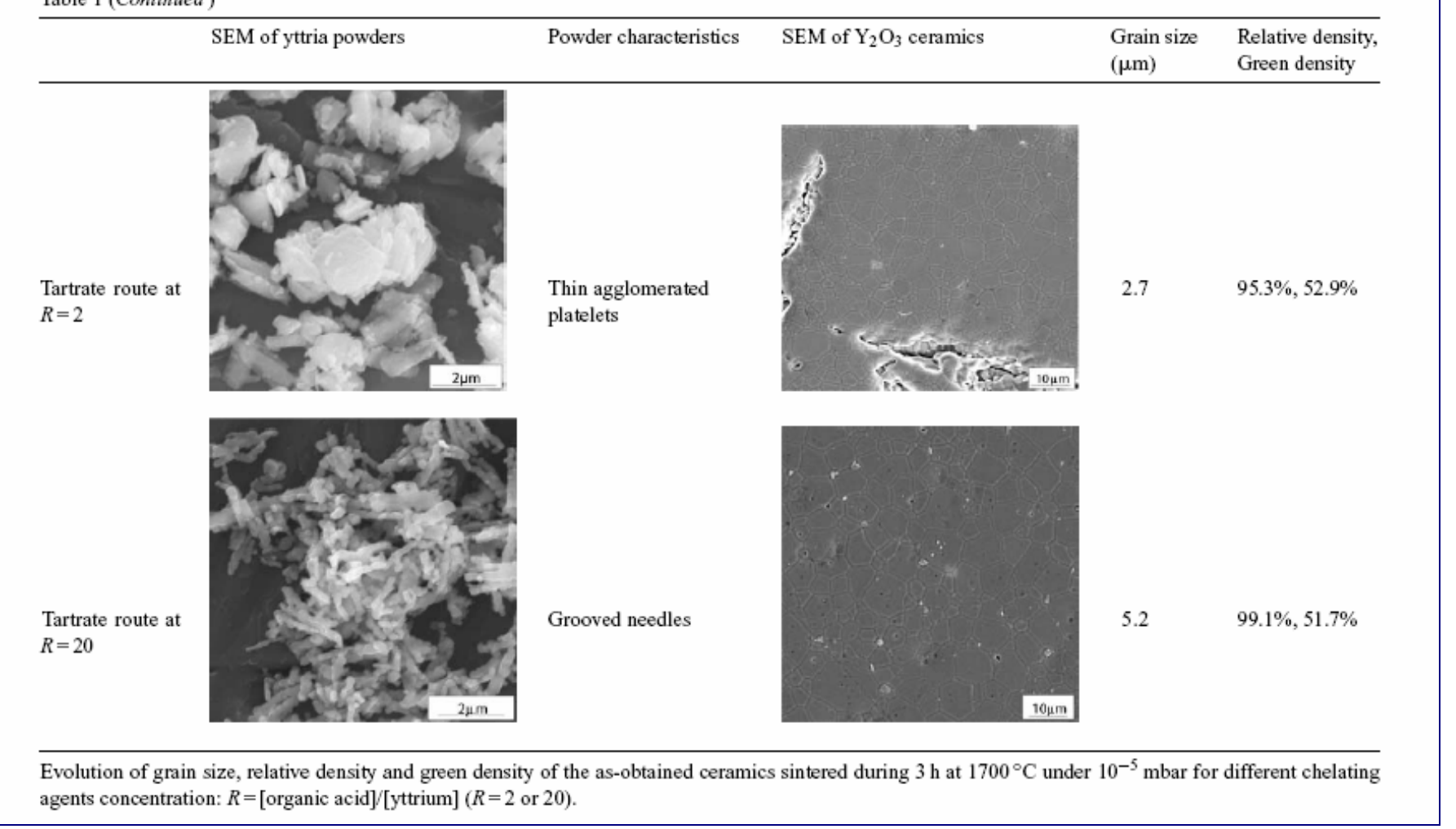

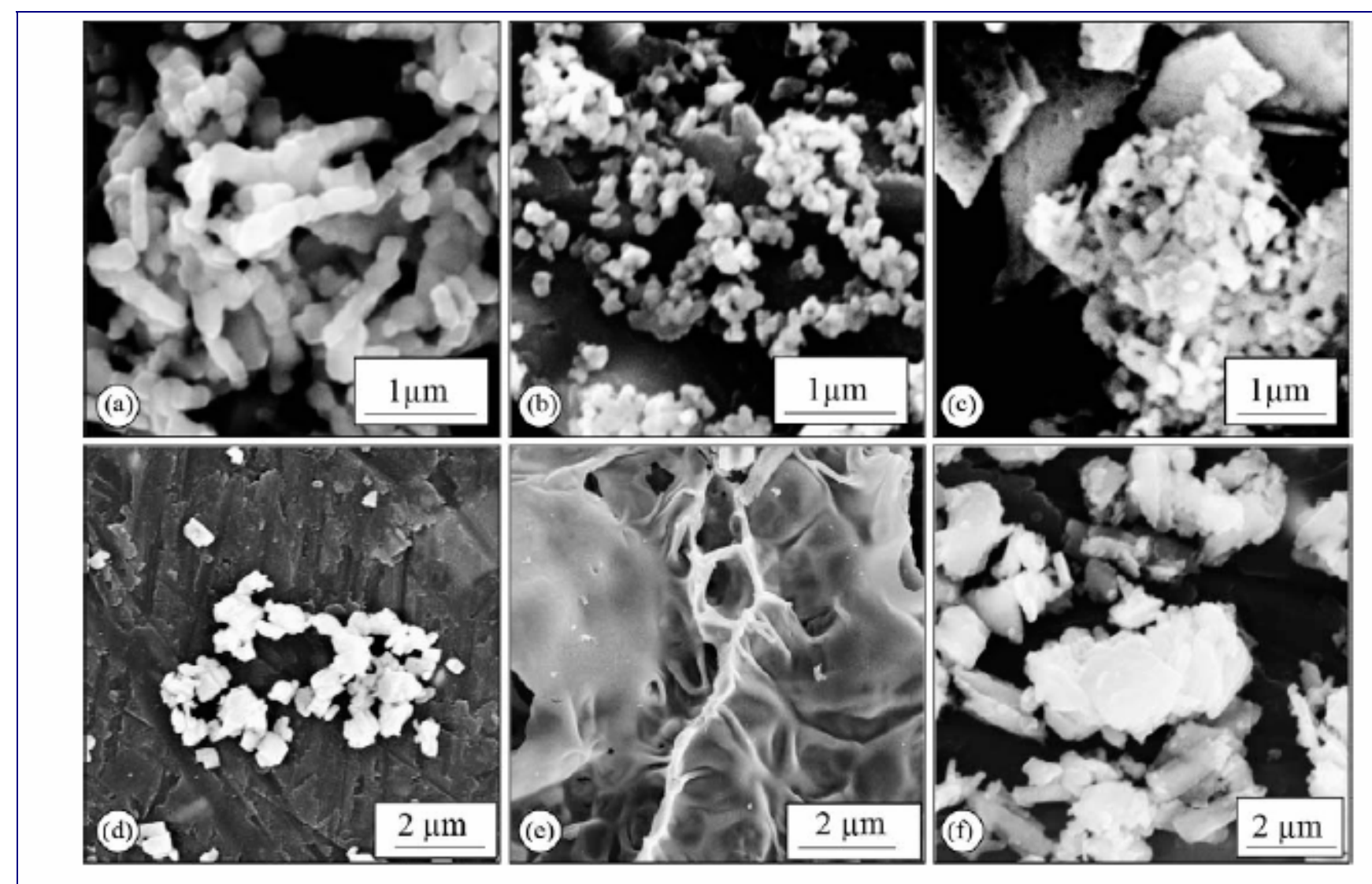

Fig. 1. Different morphologies of yttria powders before sintering. 


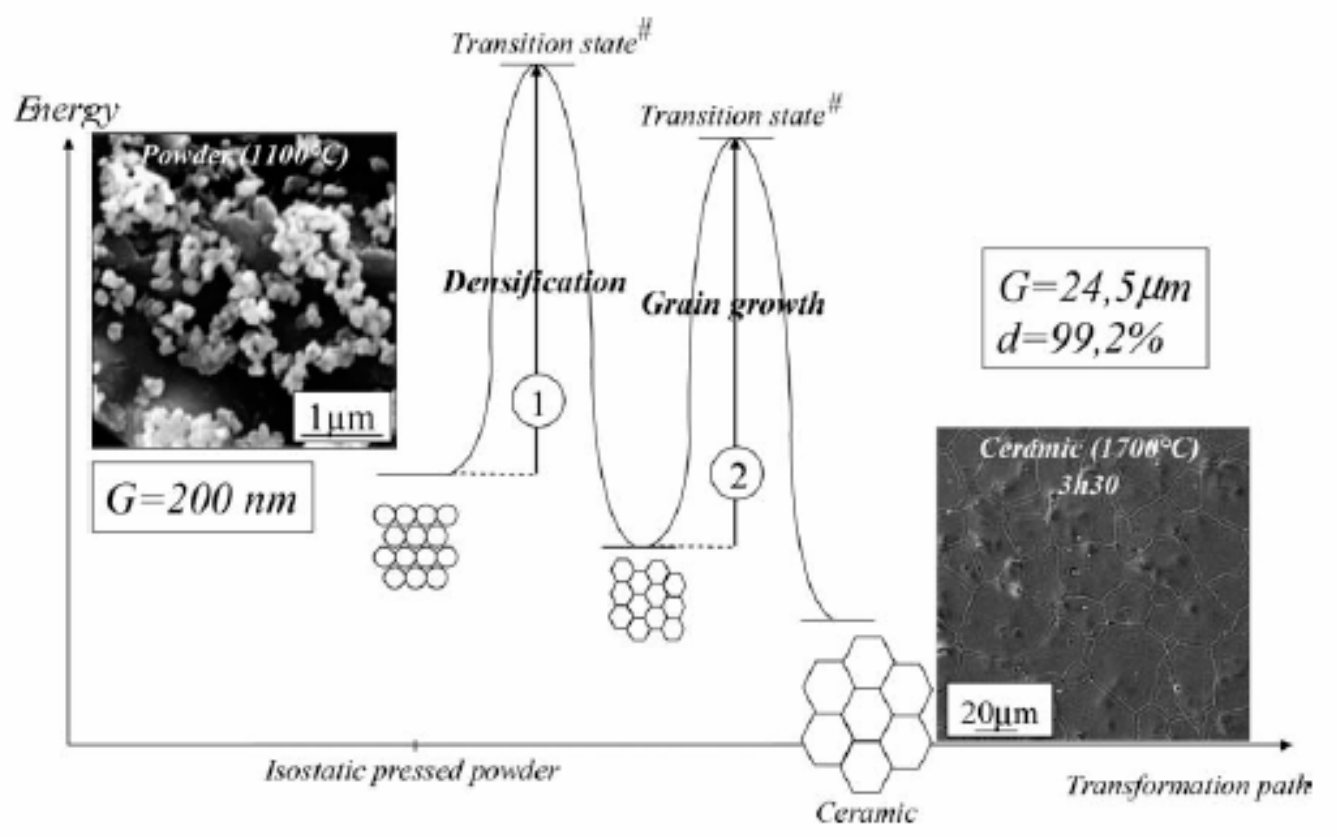

Fig. 2. Evolution of the energy of a system made up of spheres during the sintering process.

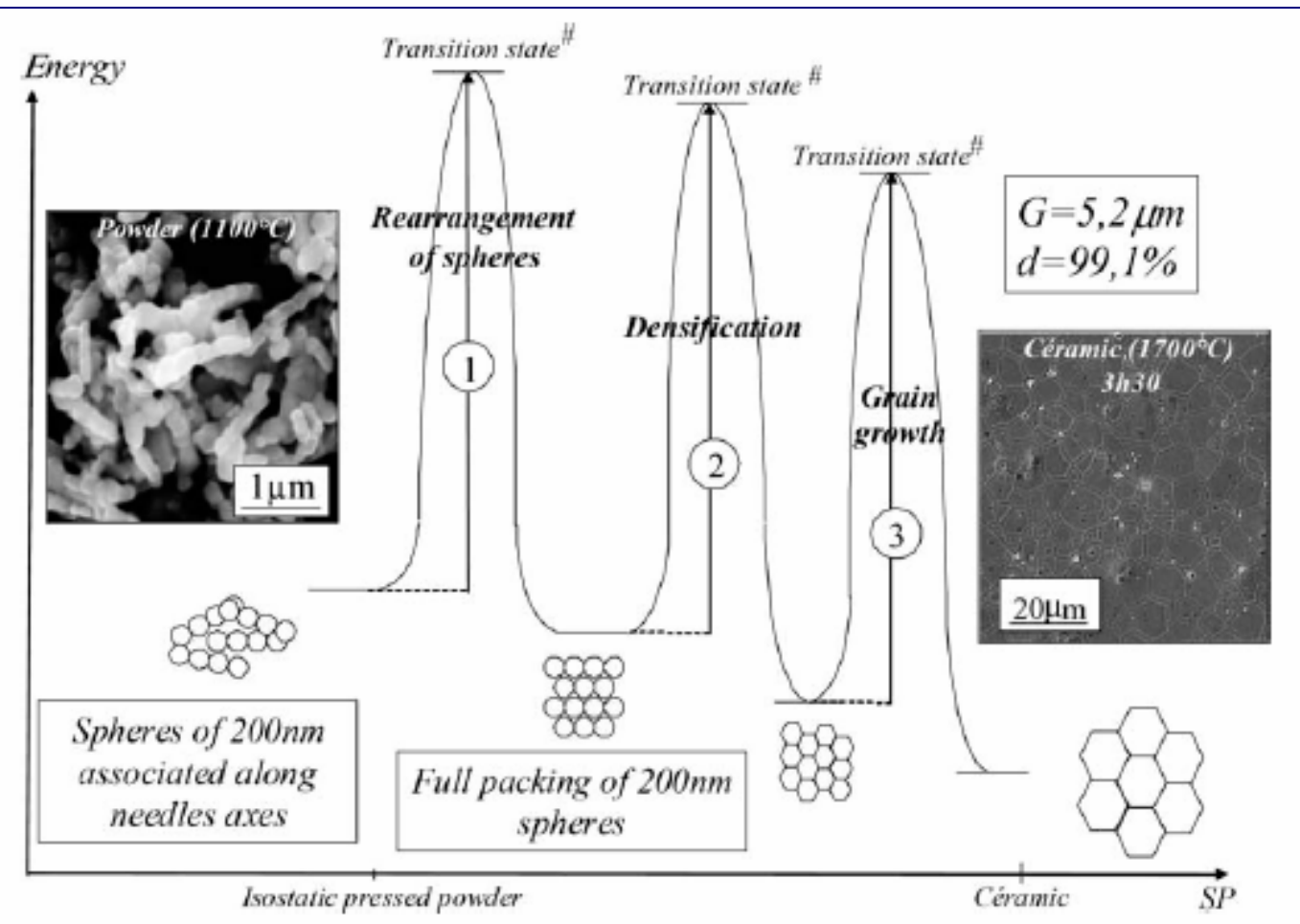

Fig. 3. Evolution of the energy of a system made up of grooved needles during the sintering process. 


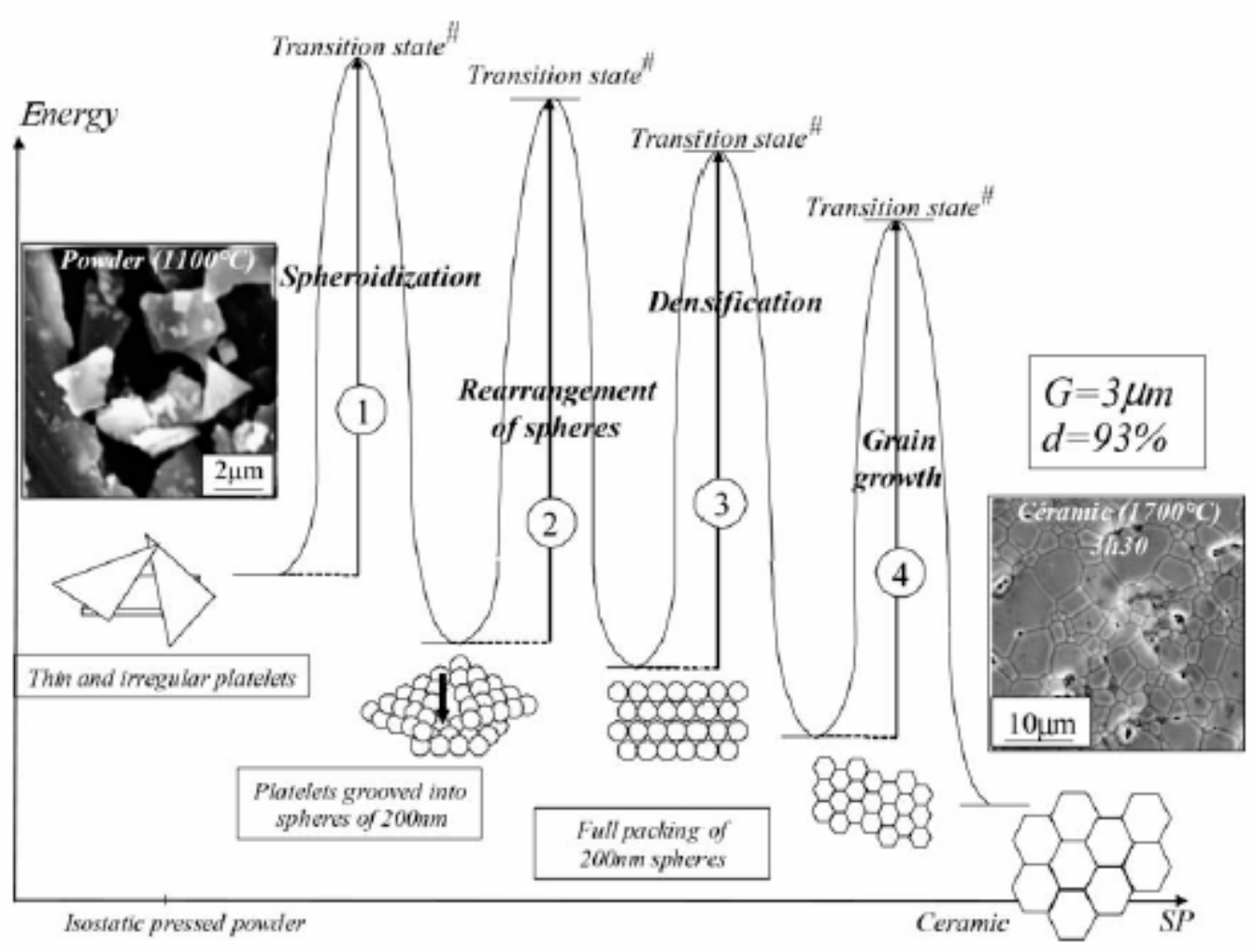

Fig. 4. Evolution of the energy of a system made up of thin and irregular platelets during the sintering process.
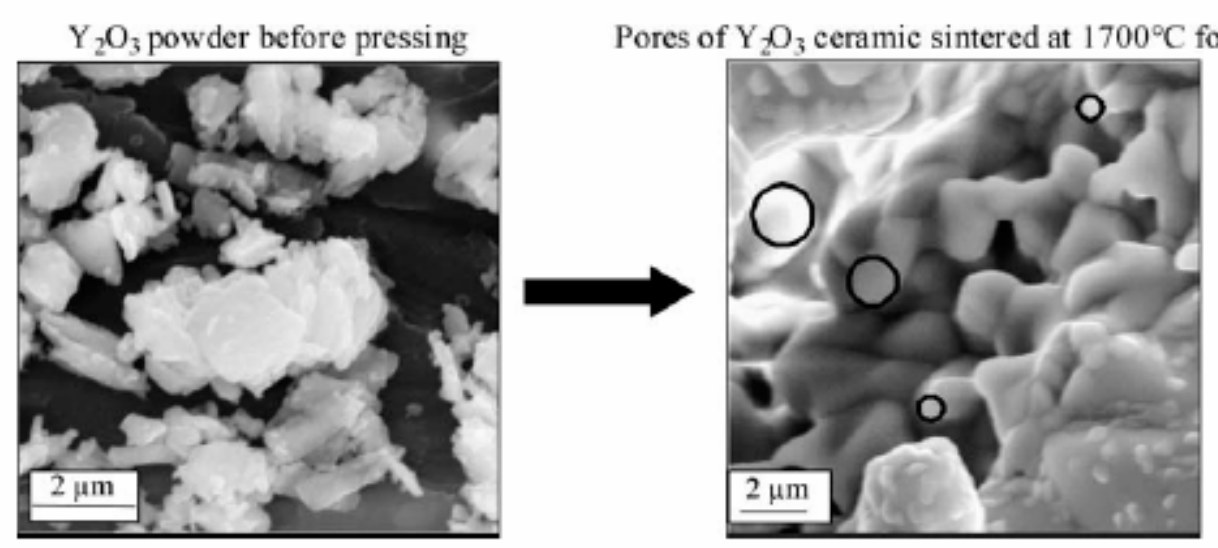

Fig. 5. Spheroidization in the pores observed after $3 \mathrm{~h}$ sintering at $1700^{\circ} \mathrm{C}$ under vacuum. 